\title{
Mechanism of tumor-derived extracellular vesicles in regulating renal cell carcinoma progression by the delivery of MALAT1
}

\author{
CHENGLUO JIN $^{1 *}$, LINMEI SHI ${ }^{2 *}$, KUNLUN LI $^{1 *}$, WEI LIU ${ }^{1}$, YU QIU $^{1}$, YAKUN ZHAO $^{1}$, \\ BAI ZHAO $^{1}$, ZHEXUN LI ${ }^{1}$, YIFEI LI ${ }^{1}$ and QINGGUO ZHU ${ }^{1}$ \\ ${ }^{1}$ Department of Urology, The Second Affiliated Hospital, Harbin Medical University; ${ }^{2}$ School of Health Management, \\ Harbin Medical University, Nangang, Harbin, Heilongjiang 150086, P.R. China
}

Received August 12, 2020; Accepted April 28, 2021

DOI: $10.3892 / o r .2021 .8138$

\begin{abstract}
Renal cell carcinoma (RCC) is a major healthcare burden globally. Tumor-derived extracellular vesicles (EVs) contribute to the formation of a pro-metastatic microenvironment. In the present study, we explored the role and mechanism of RCC cell 786-O-derived EVs (786-O-EVs) in RCC. First, 786-O-EVs were extracted and identified, and EV internalization of RCC cells was observed. RCC cell malignant behaviors and long noncoding RNA (lncRNA) metastasis-associated lung adenocarcinoma transcript 1 (MALAT1) expression patterns were detected before and after 786-O-EV treatment. MALAT1 was intervened to evaluate RCC cell behaviors. The downstream mechanism involving MALAT1 was predicted. In addition, the relationship among MALAT1, transcription factor CP2 like 1 (TFCP2L1) and ETS proto-oncogene 1, transcription factor (ETS1) was analyzed. TFCP2L1 expression patterns were measured after 786-O-EV exposure. Tumor xenograft formation assay and lung metastasis model were adopted to verify the role of 786-O-EVs in vivo in RCC. It was found that 786-O-EVs could be internalized by RCC cells. 786-O-EVs promoted RCC cell malignant behaviors, accompanied by elevated MALAT1 expression levels. The 786-O-EVs with MALAT1 knockdown attenuated the promotive effect of sole 786-O-EVs on RCC cells. MALAT1 located ETS1 in the TFCP2L1 promoter and negatively regulated TFCP2L1, and ETS1 protein could specifically bind to MALAT1. 786-O-EVs
\end{abstract}

Correspondence to: Dr Chengluo Jin, Department of Urology, The Second Affiliated Hospital, Harbin Medical University, 148 Baojian Road, Nangang, Harbin, Heilongjiang 150086, P.R. China

E-mail: jc10114@163.com

Dr Linmei Shi, School of Health Management, Harbin Medical University, Nangang, Harbin, Heilongjiang 150086, P.R. China E-mail: is6434qytq8472@163.com

*Contributed equally

Key words: renal cell carcinoma, extracellular vesicles, lncRNA MALAT1, ETS1, TFCP2L1, migration, invasion enhanced the binding of ETS1 and the TFCP2L1 promoter and decreased TFCP2L1 expression. In vivo, 786-O-EVs promoted tumor growth and RCC lung metastasis, which was suppressed following inhibition of MALAT1. Our findings indicated that 786-O-EVs promoted RCC invasion and metastasis by transporting MALAT1 to promote the binding of transcription factor ETS1 and TFCP2L1 promoter.

\section{Introduction}

Renal cell carcinoma (RCC) is regarded as one of the most common malignancies of the genitourinary system, with an increasing incidence rate in the United States (1). RCC is generally rare in children and young adults as it primarily manifested in the elderly $(2,3)$. Some of the contributing factors of RCC include hypertension, smoking, familial syndromes, with an unhealthy lifestyle and dietary habits (4). RCC has multiple subtypes, with clear cell RCC (ccRCC) as the most prevalent type with $85 \%$ proportion among other subtypes (5). Chest metastasis is a frequent finding in RCC, however lung metastasis is the most common form of distant metastasis (6). Additionally, RCC is habitually asymptomatic until the diagnosis of metastasis by medical intervention. With expanding alternative treatment options, surgical intervention persists as the gold standard for the treatment of $\operatorname{RCC}(7,8)$. Therefore, we sought to explore new and reliable protocols for RCC therapy.

Extracellular vesicles (EVs), fundamentally recognized as the nanoscale tools composed of exosomes and microvesicles for intrinsic intercellular communication, are active contributors in diverse physiological processes, including regulation of tumor development (9). A recent study ascertained the vital functionality of EVs in RCC initiation and progression (10). Tumor-derived EVs are the focus of increased research due to their ability to facilitate tumorigenesis and thus are being adopted as promising markers for tumor treatment, including $\operatorname{RCC}(11,12)$.

Furthermore, the role of EVs in mediating cell-to-cell communication and facilitating tumorigenesis is achieved via transfer of multiple RNAs, including long noncoding RNAs (lncRNAs) (13). Accumulating evidence has validated lncRNAs as crucial in regulating diverse cancers, including RCC $(14,15)$. Metastasis-associated lung adenocarcinoma transcript 1 (MALAT1) has been validated as a potent marker 
for multiple human cancers accounting for its abnormal upregulation as an indicator of aggravated pathological alterations in cancerous organs (16). MALAT1 shows an aberrantly high expression and promotes cell malignant biological behaviors in RCC (17). Furthermore, as previously evidenced, MALAT1 can be diffused by the epithelial ovarian cancer cell-secreted exosomes to the target recipient cells to promote cancer angiogenesis (18). However, the mutual action of tumor-derived EVs and MALAT1 in RCC has not yet been explored.

Therefore, we hypothesized that tumor-derived EVs may play a role in RCC with the involvement of lncRNA MALAT1. Consequently, we performed an array of histological and molecular experiments to identify the interaction between RCC cell-derived EVs and MALAT1, and to explore the relative regulatory mechanism, in an attempt to identify novel therapies against RCC.

\section{Materials and methods}

Extraction and identification of EVs. 786-O cells [American Type Culture Collection (ATCC)] underwent a 72-h culture using Dulbecco's modified Eagle's medium (DMEM) containing $10 \%$ EV-free fetal bovine serum (FBS) (Thermo Fisher Scientific, Inc.) and $1 \%$ penicillin streptomycin. The supernatant was collected and subjected to 5-min (at 1,100 x g) and then $30-\mathrm{min}$ (at 40,000 $\mathrm{x}$ g) centrifugation regimens to eliminate the cells and cellular debris, and large vesicles, respectively. After filtration of the supernatant using a $0.2-\mu \mathrm{m}$ filter and centrifugation (at $1,000,000 \mathrm{xg}$ ) for $2 \mathrm{~h}$, the EVs underwent a phosphate-buffered saline (PBS) rinse and a 2-h regimen of centrifugation (at 100,000 x g), followed by resuspension in PBS. An EV isolation reagent (Thermo Fisher Scientific, Inc.) was used for EV isolation. Nanoparticle tracking analysis (NTA) was applied for EV analysis using the NanoSight NS300 (Malvern Panalytical Co., Ltd.). The obtained EVs were allocated into the EV group and the blank group [supplemented with $1 \mu \mathrm{M}$ GW4869 (MedChemExpress Co., Ltd.)] (19). The small-interfering (si)-MALAT1-1, si-MALAT1-2 and si-negative control (NC) (Shanghai GenePharma Co., Ltd.) were transfected into the 786-O cells, respectively, using Lipofectamine 2000 (Invitrogen; Thermo Fisher Scientific, Inc.) to attain a final transfection concentration of $50 \mathrm{nM}$. The EVs (EVs/si-MALAT1-1, EVs/si-MALAT1-2 and EVs/si-NC) were extracted respectively by ultracentrifugation and identified with the preceding protocol. The morphology of the EVs was observed under transmission electron microscopy (TEM). The protein determination by the bicinchoninic acid (BCA) method was performed on all the aforementioned EVs and the result was $2.2 \mathrm{mg} / \mathrm{ml}$. Subsequent experiments were conducted using the protein concentration as the concentration of EVs.

Bioinformatics analysis. The lncDisease database (http://www.cuilab.cn/lncrnadisease) was applied to search for IncRNAs associated with RCC. The expression of the candidate IncRNAs in the tumor-EVs was searched through the exoRBase database (http://www.exorbase.org/). The lncMAP database (http://bio-bigdata.hrbmu.edu.cn/LncMAP/) was used to predict the downstream regulatory transcription factors in kidney renal clear cell carcinoma (KIRC) and kidney renal papillary cell carcinoma (KIRP). Next, the genes related to RCC were searched through the DisGeNET database (https://www.disgenet.org/). Gene interaction network diagram was constructed using the STRING database (https://string-db.org/) and Cytoscape V3.7.1 software (www. cytoscape.org). RCC microarray GSE16441 (20) including 17 normal samples and 17 RCC samples was obtained from the GEO database (https://www.ncbi.nlm.nih.gov/geo/). With the normal samples as the control, the R language 'limma' package (21) was applied for differential analysis. TheP-value was corrected using the false discovery rate (FDR) method, and $\mid \log \mathrm{FCl}>2$ and $\mathrm{FDR}<0.05$ were used as the screening criteria for the differentially expressed genes. JASPAR database (http://jaspar.genereg.net/) was used to predict the E26 transformation specific-1 (ETS1) downstream regulatory genes, and to obtain the existing binding site between transcription factor CP2-like 1 (TFCP2L1) promoter and ETS1.

Cell culture and grouping. RCC cell lines A498 and ACHN supplied by ATCC were cultured in minimal essential medium (MEM; Gibco Co.; Thermo Fisher Scientific, Inc.) with $10 \%$ FBS (Gibco; Thermo Fisher Scientific, Inc.) in an incubator at $37^{\circ} \mathrm{C}$ under saturated conditions with $5 \% \mathrm{CO}_{2}$. The A498 and ACHN cells were co-cultured with $10 \mu \mathrm{g} / \mathrm{ml}$ of the above-mentioned 786-O-EVs (22) for $24 \mathrm{~h}$, grouped and named similarly as the aforementioned EVs.

PKH26 fluorescence labeling. The 786-O-EVs were labeled by a PKH26 red fluorescence labeling kit (Sigma-Aldrich; Merck $\mathrm{KGaA}$ ) in compliance with the provided instructions. Briefly, the EVs underwent a 4-min incubation regimen with the PKH26 dye, which was terminated by the addition of EV-free FBS. The EVs were rinsed three times and the excess PKH26 dye was removed with an Amicon Ultra-4 (100-kDa, Merck Millipore Corp.), followed by a 4-h incubation regimen with the RCC cells. The internalization of EVs by RCC cells was observed under confocal microscopy.

Quantitative real-time polymerase chain reaction ( $q P C R)$. The total RNA content from the cells in each group was extracted using the TRIzol reagent (Invitrogen; Thermo Fisher Scientific, Inc.), and the RNA concentration was determined using a NanoDrop 2000 (Thermo Fisher Scientific, Inc.). The PrimeScript RT reagent kit and gDNA Eraser kit (Takara Bio Inc.) were used to transcribe the total RNA (1 mg) content into cDNA. Genomic DNA termination was induced by the addition of 5X DNA Eraser buffer and gDNA Eraser at $42^{\circ} \mathrm{C}$ for $2 \mathrm{~min}$. Next, the cDNA was synthesized with the reaction condition as $37^{\circ} \mathrm{C}$ for $15 \mathrm{~min}$ and $85^{\circ} \mathrm{C}$ for $5 \mathrm{sec}$. Next, qPCR was conducted using a SYBR Premix Ex Taq (Tli RNaseH Plus) kit (Takara) on a ABI 7500 real-time PCR instrument (Thermo Fisher Scientific, Inc.). Data of each gene were analyzed based on the $2^{-\Delta \Delta \mathrm{Cq}}$ method (23). The primer sequences are listed in Table I.

Western blot analysis (WB). The total protein content was extracted from cells in each group, and the protein concentration was measured using a BCA kit (Thermo Fisher Scientific, Inc.). Total proteins (30 mg) underwent 35-min (at $80 \mathrm{~V}$ ) and 45-min (at $120 \mathrm{~V}$ ) polyacrylamide gel electrophoresis successively. Next, the proteins were loaded onto polyvinylidene fluoride membranes (Amersham Pharmacia), blocked for $1 \mathrm{~h}$ using 5\% skim milk, and probed with the corresponding 
Table I. Primer sequences for qPCR.

\begin{tabular}{ll}
\hline Gene & \multicolumn{1}{c}{ Primer } \\
\hline MALAT1 & F: 5'-GGGGCAGTAGTGTAGAGA-3' \\
& R: 5'-CAGTGCGTGTCGTGGAGT-3' \\
U6 & F: 5'-CTCGCTTCGGCAGCACA-3' \\
& R: 5'-GTGTCGTGGAGTCGGCAA-3' \\
TFCP2L1 & F: 5'-AGGTGCTGACCTCCTGAAGA-3' \\
& R: 5'-GTTTTGCTCCAGCTCCTGAC-3' \\
ETS1 & F: 5'-CATGCTTTTCGTTTGACACCC-3' \\
& R: 5'-CTTTGCTTCCACCCGCCCCCC-3' \\
GAPDH & F: 5'-ACCAGGTATCTGCTGGTTG-3' \\
& R: 5'-TAACCATGATGTCAGCGTGGT-3'
\end{tabular}

F, forward; R, reverse; MALAT1, metastasis-associated lung adenocarcinoma transcript 1 ; TFCP $2 L 1$, transcription factor CP2 like 1; ETS1, ETS proto-oncogene 1; GAPDH, glyceraldehyde 3-phosphate dehydrogenase.

primary antibodies [cluster of differentiation (CD)63 (dilution 1:1,000, ab134045), CD81 (dilution 1:1,000, ab109201) and TFCP2L1 (dilution 1:1,000, ab140197) (all from Abcam Inc.)] at $4^{\circ} \mathrm{C}$. Following 3 rinses (10 min each) with PBS and $0.1 \%$ Tween-20 (PBST), the membranes underwent a 1-h incubation regimen with the horseradish peroxidase-labeled goat anti-rabbit immunoglobulin $\mathrm{G}$ ( $\mathrm{IgG}) \mathrm{H} \& \mathrm{~L}$ (dilution 1:2,000, ab205718; Abcam Inc.), and rinsed 3 times with PBST (10 min each). An optical illuminator (General Electric) was utilized for membrane visualization. Image-Pro Plus 6.0 (Media Cybernetics) was applied for protein band gray value analysis.

Cell Counting Kit-8 (CCK-8) assay. RCC cell proliferation was detected using the CCK-8 kit (Dojindo Molecular Technologies) in strict accordance with the provided instructions. RCC cells were seeded into 96-well plates and each well was supplemented with EVs based on cell grouping and incubation for $24 \mathrm{~h}$. Then approximately $10 \mu \mathrm{l} \mathrm{CCK}-8$ reagent was added to each well for a 2 -h regimen of incubation at $37^{\circ} \mathrm{C}$. The optical density (OD) at the wavelength of $490 \mathrm{~nm}$ was determined using a microplate reader (Thermo Fisher Scientific, Inc.). Relative cell viability of each group was determined.

Transwell assays. RCC cell invasion was assessed according to the passage of the number of transfected cells through Transwell chambers (8-micron chamber; Corning Inc., Life Sciences). The Transwell chambers were pre-coated with $100 \mu 1$ Matrigel (BD Biosciences) at $37^{\circ} \mathrm{C}$ for $5 \mathrm{~h}$ until gelling was visible. Next, $1 \times 10^{5} \mathrm{~A} 498$ and ACHN cells that underwent a 24-h starvation in $500 \mu \mathrm{l}$ serum-free MEM medium were seeded in the apical chamber, respectively (3 technical replicates for each group). The basolateral chamber was filled with $700 \mu \mathrm{l}$ MEM with $10 \%$ FBS. Following a 48-h incubation at $37^{\circ} \mathrm{C}$, Matrigel and non-invaded cells were removed from the upper surface of the filters. Cells that adhered to the lower surface of filters were fixed using $4 \%$ paraformaldehyde and stained with crystal violet. Finally, the cells were counted and photographed using a Nikon digital camera (magnification, $\mathrm{x} 100)$. Three independent experiments were repeatedly set. The method for the migration experiment was the same as that of the invasion experiment, without utilization of Matrigel.

Immunofluorescence. The cells were seeded on 24-well plates and received different treatments. Next, the cells were fixed for $15 \mathrm{~min}$ in $4 \%$ paraformaldehyde, permeated for $10 \mathrm{~min}$ with PBS containing $0.1 \%$ Triton X-100, and sealed for $30 \mathrm{~min}$ using the $3 \%$ plasma blocking solution (all performed at room temperature). Subsequently, the cells were subjected to overnight incubation at $4^{\circ} \mathrm{C}$ with the diluted primary antibodies E-cadherin (dilution 1/200, ab1416, Abcam) and N-cadherin (dilution 1/200, ab76057, Abcam), followed by a 1-h incubation regimen with the secondary antibody goat anti-mouse IgG H\&L (Alexa Fluor ${ }^{\circledR}$ 488) (dilution 1/200, ab150117, Abcam) in conditions devoid of light at room temperature. Finally, 4',6-diamidino-2-phenylindole (DAPI) was added for nuclear staining. After resting at room temperature for $1 \mathrm{~min}$, the cells were observed under a fluorescence microscope (Olympus Optical Co., Ltd.).

Fluorescence in situ hybridization (FISH). MALAT1 subcellular localization was observed using FISH assay in strict accordance with the provided instructions of the Ribo ${ }^{\mathrm{TM}}$ lncRNA FISH Probe Mix (Red) (C10920, RiboBio Co., Ltd.). The cells $\left(6 \times 10^{4}\right.$ cells/well $)$ were seeded into 24 -well plates. Upon achieving 60-70\% confluence, the cells were fixed using $4 \%$ paraformaldehyde, rinsed and permeabilized. The plates were sealed using the pre-hybridization solution. After elimination of the pre-hybridization solution, the cells were subjected to overnight hybridization at $37^{\circ} \mathrm{C}$ using the probe hybridization solution supplemented with anti-MALAT1 nucleotide (Wuhan Genecreate Bioengineering Co., Ltd.) in conditions devoid of light. Next, the cells were eluted, stained with DAPI, rinsed, and fixed using nail polish for observation under fluorescence microscopy (Olympus). Five different fields were selected, and the cells in these fields were observed and documented.

Dual-luciferase reporter gene assay. To explore the effect of MALAT1 on TFCP2L1 promoter activity, overexpressed (oe)-NC, oe-MALAT1, short hairpin (sh)-NC, and sh-MALAT1 were co-transfected with the TFCP2L1-2 kb luciferase reporter plasmid respectively into $293 \mathrm{~T}$ cells. After $48 \mathrm{~h}$ of transfection, the cells were collected and lysed. The dual-luciferase reporter gene assay was performed using a luciferase assay kit (K801-200, BioVision Inc.) and a dual-luciferase reporter gene analysis system (Promega Corp.). Renilla luciferase was adopted for internal reference. The activation degree of the target reporter gene was measured using the ratio of the relative unit of firefly luciferase to that of Renilla luciferase.

RNA immunoprecipitation (RIP). A RIP kit (Millipore Corp.) was utilized to assess the binding of MALAT1 to the ETS1 protein. The cells were lysed in an ice bath, and then subjected to centrifugation. Next, the supernatant was collected. The cell extract was incubated with the corresponding antibody for co-precipitation. Following a rinse and re-suspension in RIP wash buffer, the magnetic beads were supplemented with the 
appropriate antibody for binding. After a rinse, the magnetic bead-antibody complex was resuspended in RIP wash buffer, incubated overnight at $4^{\circ} \mathrm{C}$ with the cell extract, and harvested. The RNA content was extracted from the sample for subsequent PCR detection after detachment with proteinase $\mathrm{K}$. The antibody used above was ETS1 (dilution 1:200, ab225868, Abcam), with IgG (dilution 1:100, ab172730, Abcam) as the control.

RNA pull-down. Biotinylated RNA sequence probe in the TFCP2L1 promoter region and its negative control (NC) probe were dissolved in washing/binding buffer at room temperature and incubated with the streptavidin-coupled magnetic beads for $2 \mathrm{~h}$. Then, the cell lysate was added and incubated for $2 \mathrm{~h}$. The protein complex conjugated to magnetic beads was washed. ETS1 content in the complex was determined by western blot analysis.

Ethics statement. The experimental procedures were approved by the Ethics Committee of The Second Affiliated Hospital of Harbin Medical University. Significant efforts were made in order to minimize both the number of animals used as well as their respective suffering.

Subcutaneous tumorigenesis in mice and lung metastasis model establishment. A total of $48 \mathrm{BALB} / \mathrm{C}$ nude mice (male, aged 4 weeks, weighing 15-18 g; 6 each per group) used for animal experiments and xenograft collection were supplied by Beijing Wantai BioPharm Co., Ltd. [Beijing, China; SYXK (Jing) 2017-0041]. Each nude mouse was housed in a separate cage and provided with free access to food and water. All mice were fed with clean grade maintenance feed and kept at a temperature of $26-28^{\circ} \mathrm{C}$ and humidity at 40-60\% with 10/14 h light/dark cycle. The nude mice were subcutaneously injected with the 786-O cell suspension $\left(5 \times 10^{6}\right.$ cells $)$, and treated with the blank, EVs, EVs/si-NC or EVs/si-MALAT1-1 (20 nM) using Entranster ${ }^{\mathrm{TM}}$-in vivo Transfection Reagent (Engreen Biosystem Co., Ltd.) after acquiring a tumor volume greater than $100 \mathrm{~mm}^{3}(\mathrm{n}=6)$ in each nude mouse. Xenografts were determined weekly. The nude mice were euthanized via intraperitoneal injection of $800 \mathrm{mg} / \mathrm{kg}$ pentobarbital sodium after 5 weeks and the tumor was dissected and weighed. The Ki67-positive rate was observed and counted.

The grouping of the lung metastasis model was the same as the subcutaneous tumorigenesis. Then, 6 nude mice from each group were injected with the ACHN cells $\left(2 \times 10^{6}\right.$ cells) via tail vein. After 42 days, the mice were euthanized by an intraperitoneal injection with $800 \mathrm{mg} / \mathrm{kg}$ pentobarbital sodium. The lung tissues were extracted and stained using hematoxylin and eosin (H\&E) (JRDUN Biotechnology Co., Ltd.).

\section{Histological analysis}

Immunohistochemical analysis. The removed tumor sample was fixed using 4\% paraformaldehyde and embedded in paraffin, which was then sectioned, dewaxed, and hydrated independently. The Ki67 antibody (dilution 1:500, ab15580, Abcam) was used for immunohistochemical staining. The Ki67-positive rate was observed and counted under a microscope (BX53M, Olympus; magnification, x200).
$48 H \& E$ staining. The extracted lung tissue was fixed using $4 \%$ paraformaldehyde and embedded in paraffin. Then, the tissue was sectioned, dewaxed and hydrated independently. The sections were stained using the hematoxylin solution for $10 \mathrm{~min}$ and decolorized in 70 and $90 \%$ ethanol after a rinse with distilled water. Subsequently, the sections were stained with the eosin solution for 2-3 min, dehydrated with pure ethanol, cleared with xylene, sealed with neutral gum and then observed under a microscope (BX53M, Olympus; magnification, $\mathrm{x} 200)$.

Statistical analysis. SPSS 21.0 (IBM Corp.) software was used for data analysis. The experimental data were in normal distribution as verified by Kolmogorov-Smirnov test, and expressed as mean \pm standard deviation. Comparison between two groups was conducted with the independent sample t-test. The comparison among groups was analyzed using one-way analysis of variance (ANOVA), followed by the Tukey's multiple comparisons test. The P-value was obtained from a two-sided test and a value of $\mathrm{P}<0.05$ was indicative of statistical significance.

\section{Results}

786-O-EVs were successfully extracted. After a 48-h culture regimen of 786-O cells in EV-free FBS, the EVs were separated by ultracentrifugation. NTA confirmed the size of the EVs as ranging between 30-150 nm (Fig. 1A). Consistently, TEM revealed that the EVs were oval with a diameter of about $100 \mathrm{~nm}$ (Fig. 1B). Next, according to the WB results, the EV symbolic markers (CD63 and CD81) were positively expressed in the 786-O-EVs (Fig. 1C). From the aforementioned findings, the separated granules were identified as EVs.

786-O-EVs promote RCC cell invasion and migration. An existing study reported that the tumor-derived EVs elicit negative effects on habitual cellular functions (24). Therefore, we speculated that RCC cell-derived EVs may have similar effects on the biological behaviors of RCC cells. To ascertain this speculation, we labeled the EVs with $\mathrm{PKH} 26$ red fluorescence dye after which these EVs were co-cultured $\left(10 \mu \mathrm{g} / 1 \times 10^{5}\right.$ cells) with the A498 and ACHN cells for $48 \mathrm{~h}$. Under fluorescence microscopy the A498 and ACHN cells elicited red fluorescence in their cytoplasm (Fig. 2A), indicating the internalization of 786-O-EVs by the RCC cells. Next, the A498 and ACHN cell viability was investigated to explore the effect of 786-O-EVs on A498 and ACHN cell functions. CCK-8 assay verified that the A498 and ACHN cell viability increased significantly after treatment with the 786-O-EVs (Fig. 2B) (both $\mathrm{P}<0.05$ ). Meanwhile, Transwell assays revealed that 786-O-EV treatment significantly increased A498 and ACHN cell migration and invasion (Fig. 2C) (all $\mathrm{P}<0.05)$. Next, the A498 and ACHN cell epithelial mesenchymal transition (EMT) was detected by immunofluorescence, which was evidently promoted after 786-O-EV treatment (Fig. 2D). Briefly, 786-O-EVs promoted RCC cell invasion and metastasis.

786-O-EVs are the communication mechanism of MALATI in $R C C$. From the preceding results, it is evident that the 786-O-EVs promoted RCC cell invasion and metastasis. To 
A

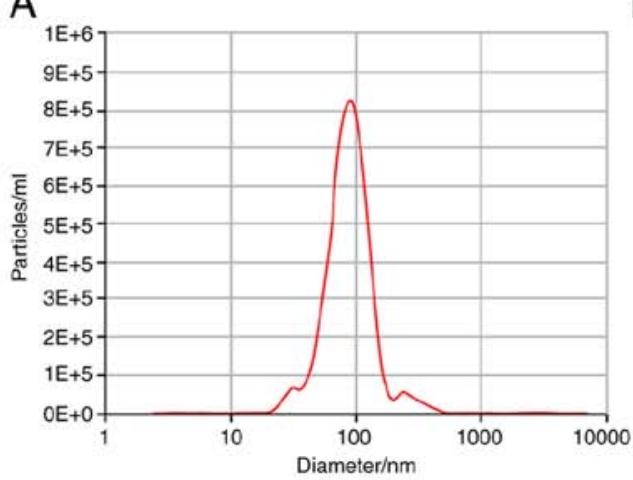

B

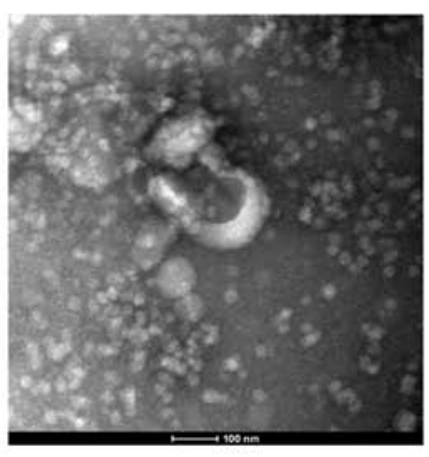

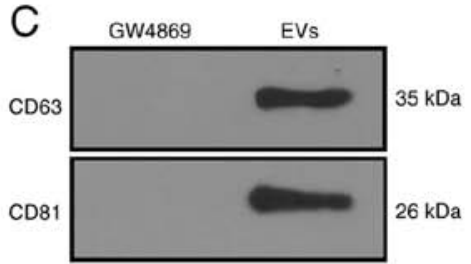

Figure 1. 786-O-EVs are successfully extracted. (A) NTA was used to analyze the size of the EV population. (B) TEM images of purified 786-O-EVs. (C) WB was used to detect the expression of EV symbolic markers. NTA, nanoparticle tracking analysis; TEM, transmission electron microscopy; EVs, extracellular vesicles.

A
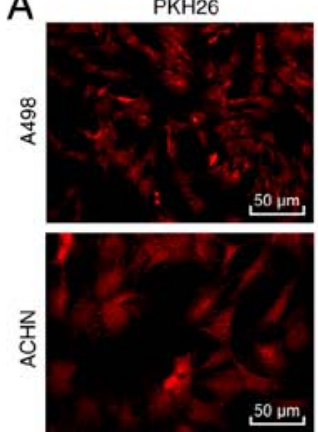

C
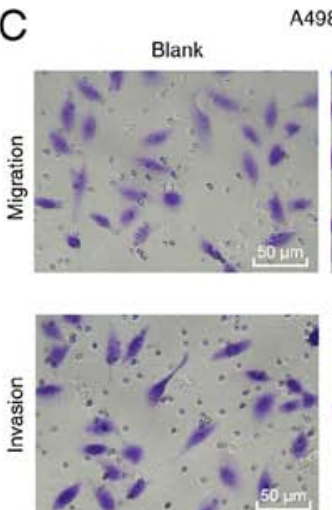

D
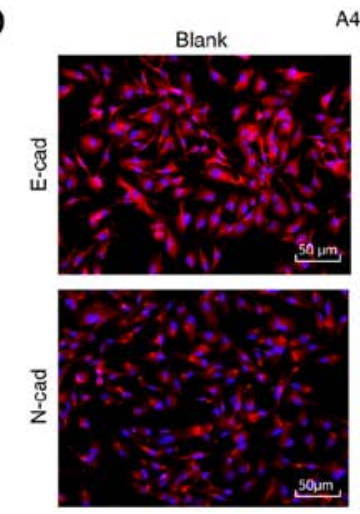

DAPI
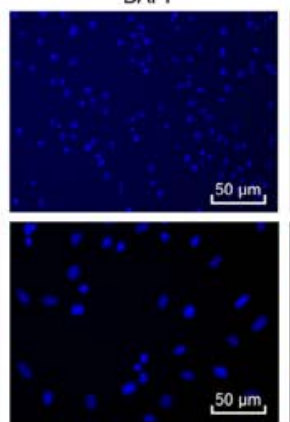

$50 \mu \mathrm{m}$
MERGE
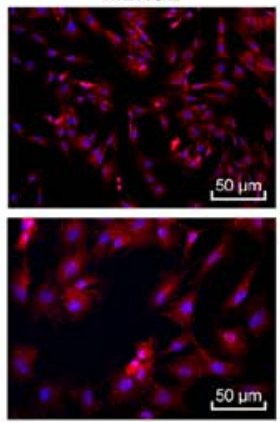

B

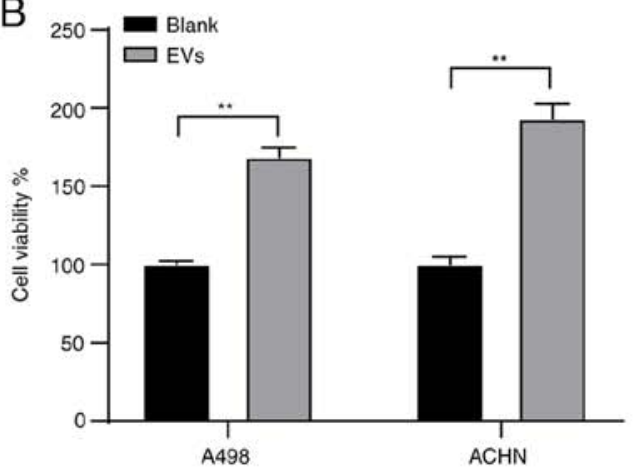

ACHN

EVs

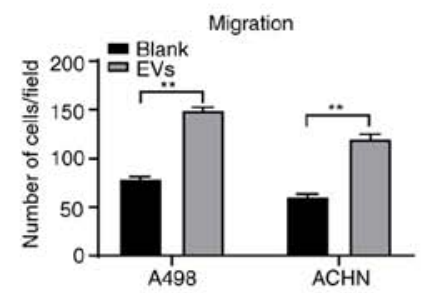

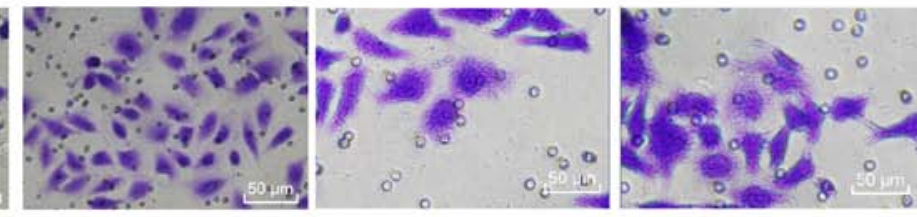

A498

$\mathrm{ACHN}$
EVs
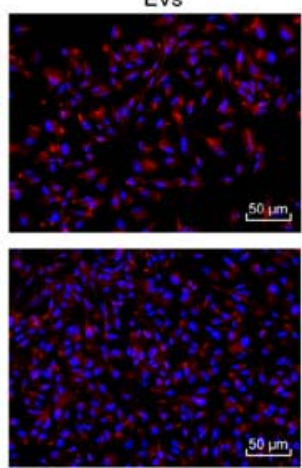
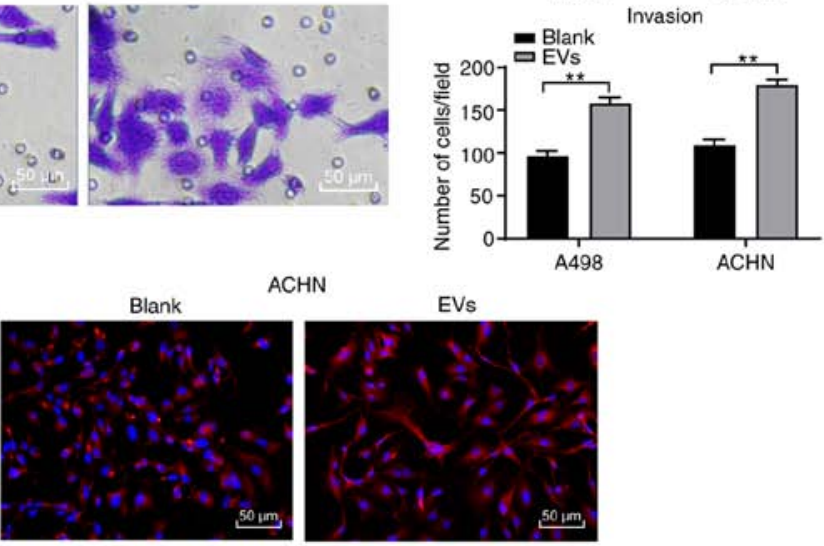

EVs

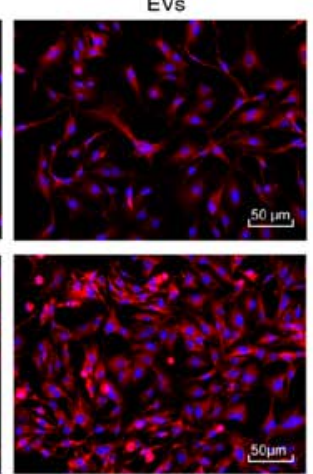

Figure 2. 786-O-EVs promote RCC cell invasion and metastasis. 786-O-EVs were incubated with A498 and ACHN cells respectively for 24 h. (A) The internalization of 786-O-EVs by A498 and ACHN cells was observed using PKH26 fluorescence labeling. (B) The viability of cells treated with 786-O-EVs was evaluated using CCK-8 assay. (C) The invasion and migration of cells were evaluated using Transwell assays. (D) The fluorescence expression of E-cadherin (E-cad) and $\mathrm{N}$-cadherin (N-cad) in A498 and ACHN cells was detected using immunofluorescence. Each experiment was repeated three times independently. Data in panels B and $\mathrm{C}$ were analyzed using independent $\mathrm{t}$-test. ${ }^{* *} \mathrm{P}<0.05$. RCC, renal cell carcinoma; EVs, extracellular vesicles. 
explore the underlying mechanism, we referred to previous studies. For instance, EVs were found to release and carry IncRNAs into the target cells to manipulate cellular functions (25). MALAT1 was also found to promote RCC cell malignant biological behaviors (26). Therefore, we searched RCC-related lncRNAs through the lncDisease database (http://www.cuilab.cn/lncrnadisease), and found the involvement of lncRNAs such as MALAT1 in the regulation of RCC (Table SI). Furthermore, the expression of these candidate lncRNAs in the EVs was searched through the exoRBase database (http://www.exorbase.org/) (Fig. 3A), which revealed that MALAT1 was expressed in various tumor-EVs, suggesting that MALAT1 may also exist in RCC cell-derived EVs. Hence, we speculated that 786-O-EVs carried MALAT1 and perhaps were internalized by the A498 and ACHN cells, thus inducing RCC cell malignant biological behaviors. MALAT1 expression pattern in the A498, ACHN and 786-O cells was determined by qPCR with no distinctive difference indicated by the results. Subsequently, the MALAT1 expression pattern in the A498 and ACHN cells before and after EV treatment was initially detected, which revealed a significant increase after EV treatment (Fig. 3B) (both $\mathrm{P}<0.01$ ). RNase treatment did not affect the MALAT1 expression pattern in the culture medium of ACHN and A498 cells; while RNase and Triton X-100 treatment showed a significant decreased MALAT1 expression pattern in the culture medium of ACHN and A498 cells (Fig. 3C) (all $\mathrm{P}<0.01)$. These results indicated that MALAT1 was membrane-encapsulated over direct release.

786-O-EVs promote RCC cell invasion and migration via transferring MALATI. From the preceding results, we established that 786-O-EVs were the communication medium of MALAT1 between RCC cells. To further validate the mechanism, we silenced the MALAT1 expression pattern in 786-O cells, and then extracted the EVs (EVs/si-MALAT1-1/2). We found that the MALAT1 expression pattern in the 786-O-EVs was significantly decreased after inhibition of MALAT1 in the 786-O cells (Fig. 4A and B). Similar reduction in the MALAT1 expression pattern was evident in RCC cells treated with 786-O-EVs after treatment with EVs/si-MALAT1-1/2 (Fig. 4C) (all $\mathrm{P}<0.01$ ). In addition, the biological behavior changes of cells in each group were evaluated. EVs/si-MALAT1-1/2 weakened the explicit effect of simple 786-O-EVs on RCC cell viability, invasion and migration and EMT capacity (Fig. 4D-F) (all $\mathrm{P}<0.01$ ). Collectively, 786-O-EVs transferred MALAT1 to induce RCC cell vitality, invasion and migration and EMT.

786-O-EVs carry MALAT1 to regulate transcription factor ETS1 and reduce TFCP2L1 activity. For a comprehensive understanding of the regulatory mechanism of MALAT1, we predicted the downstream regulatory transcription factors of MALAT1 in KIRC and KIRP through the lncMAP database (Table SII). Intersection of the predicted transcription factors in the two diseases was evidently intersected (Fig. 5A), from which four candidate transcription factors were chosen. Subsequently, the genes relevant to RCC were retrieved through the DisGeNET database (https://www.disgenet.org/) (Table SIII), where the genes with scores higher than 0.3 were selected for subsequent analyses. The interaction between the retrieved known genes and the predicted four transcription factors was analyzed, after which the gene interaction network diagram was constructed (Fig. 5B). It was evident that ETS1 had the most interactions with known genes, suggesting the superior vitality of ETS1 relative to others. In a previous work, ETS1 served as an oncogene in RCC with intimate relations with a low survival rate in a large number of RCC samples (27). Meanwhile, a RCC microarray GSE16441 was obtained through the GEO database (https://www.ncbi.nlm. nih.gov/geo/), where 406 differentially downregulated genes were identified by differential analysis of the GSE16441 chip. Concurrently, the ETS1 downstream regulatory factors were predicted through the JASPAR database (http://jaspar.genereg. net/), where the intersection between the downregulated genes in the chip and JASPAR prediction results was engaged, and five genes were identified to be present in the respective intersection (Fig. 5C). Their differential expression levels in the GSE16441 chip were further studied, where TFCP2L1 was regarded as the most notably downregulated gene (Table II). As previously highlighted, TFCP2L1 is a critical developmental transcription factor in normal kidney development (28). Meanwhile, JASPAR database predicted a number of binding sites between ETS1 and the TFCP2L1 promoter (Table SIV). Therefore, we speculated that 786-O-EVs transferred MALAT1, to subsequently regulate the transcription factor ETS1 to modulate TFCP2L1 expression, thereby affecting RCC development.

Subsequently, FISH revealed nuclear subcellular localization of MALAT1 in the cell (Fig. 5D). Dual-luciferase reporter gene assay verified that the MALAT1 overexpression remarkably reduced the TFCP2L1 promoter activity, while MALAT1 knockdown dramatically increased the TFCP2L1 promoter activity, indicating the negative regulatory effect of MALAT1 on the TFCP2L1 gene expression pattern (Fig. 5E) (both $\mathrm{P}<0.01$ ). Next, RIP was applied to further elucidate the underlying mechanism of MALAT1 to negatively regulate the TFCP2L1 gene expression pattern, and we observed that ETS1 would bind to more MALAT1 than IgG (Fig. 5F) $(\mathrm{P}<0.01)$, indicating that the ETS1 protein could specifically bind to MALAT1.RNA pull-down showed that ETS1 could bind to the TFCP2L1 promoter region (Fig. 5G). The binding of ETS1 and the TFCP2L1 promoter in the A498 and ACHN cells treated with EVs was enhanced whereas the binding of ETS1 and TFCP2L1 promoter was weakened after silencing of MALAT1 in EVs (Fig. 5H). As shown by qPCR results, 786-O-EV treatment significantly decreased the TFCP2L1 mRNA expression pattern. Following knockdown of MALAT1 in the 786-O cells, the downregulation effect of EVs on TFCP2L1 was alleviated (Fig. 5I). The above results indicated that IncRNA MALAT1 would bind to the transcription factor ETS1 and identify the fundamental complex in the sequence of TFCP2L1 promoter to inhibit transcription. Namely, MALAT1 could specifically bind to ETS1 and inhibit TFCP2L1 expression.

786-O-EVs promote $R C C$ cell growth and metastasis in vivo through MALAT1. To confirm that 786-O-EVs promote RCC cell invasion and migration through MALAT1 in vivo, we conducted tumor xenograft formation assay, and measured the tumor volume weekly for 5 weeks. We observed that 786-O-EVs had notably promoted tumor growth in vivo 

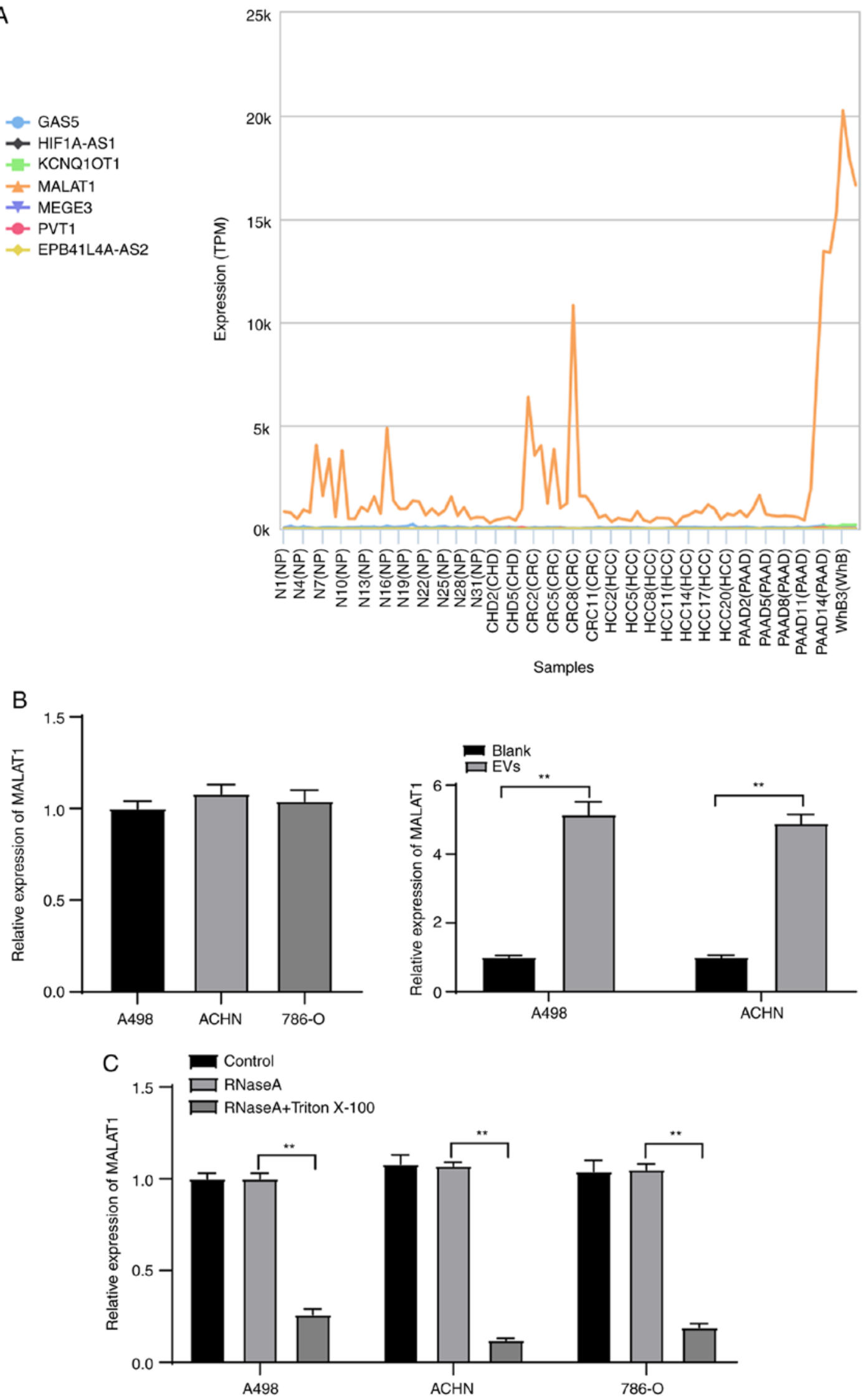

Figure 3. 786-O-EVs are the communication mechanism of MALAT1 in RCC cells. (A) The expression of candidate lncRNAs in the EVs evaluated through the exoRBase database (http://www.exorbase.org/); the abscissa represents the EVs from different tumors, and the ordinate represents the expression of different lncRNAs; the left side shows the diagram. NP, normal person; CHD, coronary heart disease; CRC, colorectal cancer; HCC, hepatocellular carcinoma; PAAD, pancreatic adenocarcinoma; WhB, whole blood. (B) Expression of MALAT1 in ACHN and A498 cells treated with 786-O-EVs was detected using qPCR. (C) Expression of MALAT1 in 786-O, ACHN and A498 cells after treatment of RNase and Triton X-100 was detected using qPCR. Each experiment was repeated three times independently. Data in panels B and C were analyzed using independent t-test or one-way ANOVA, followed by Tukey's multiple comparisons test. ${ }^{* *} \mathrm{P}<0.01$. EVs, extracellular vesicles; MALAT1, metastasis-associated lung adenocarcinoma transcript 1. 

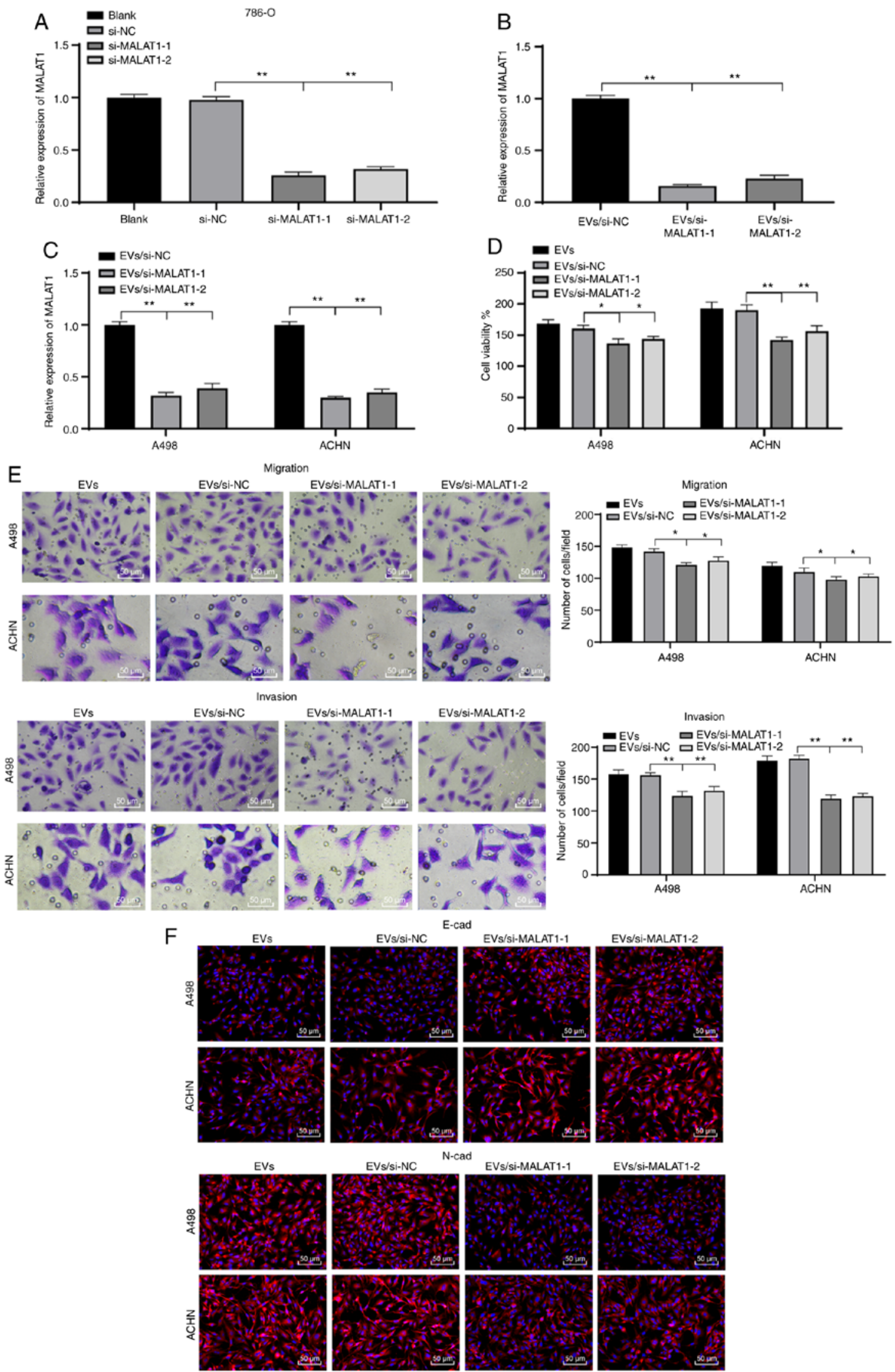

Figure 4. 786-O-EVs promote RCC cell invasion and migration via transferring MALAT1. EVs were extracted after si-MALAT1 was transfected into 786-O cells. (A) siRNA transfection efficiency of MALAT1 in 786-O cells was detected using qPCR. (B) MALAT1 expression in 786-O-EVs was detected using qPCR. (C) MALAT1 expression in ACHN and A498 cells treated with EVs was detected using qPCR. (D) CCK-8 assay was used to evaluate the viability of ACHN and A498 cells. (E) Transwell assay was used to evaluate invasion and migration ability of cells. (F) Immunofluorescence was used to detect EMT-related protein fluorescence expression in each group. Each experiment was repeated three times independently. Data in panels A and B were analyzed using one-way ANOVA, and data in panels C-E were analyzed using one-way ANOVA, followed by Tukey's multiple comparisons test. ${ }^{*} \mathrm{P}<0.05$, ${ }^{* *} \mathrm{P}<0.01$. EVs, extracellular vesicles; MALAT1, metastasis-associated lung adenocarcinoma transcript 1; RCC, renal cell carcinoma. 
A

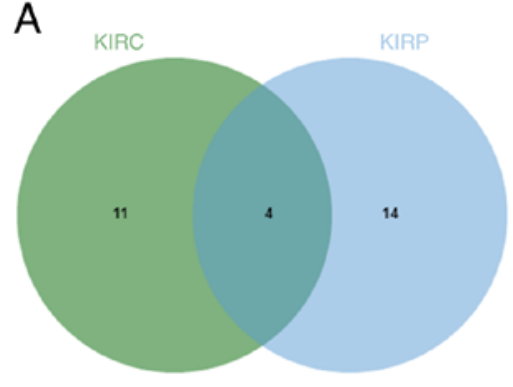

B

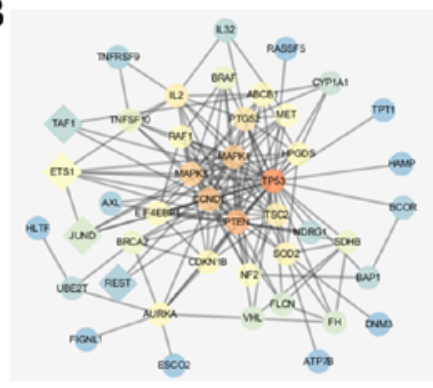

C

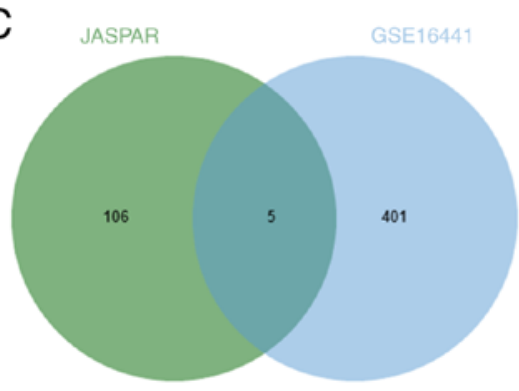

D

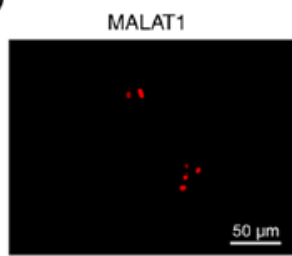

DAPI

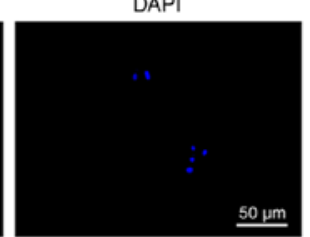

Merge

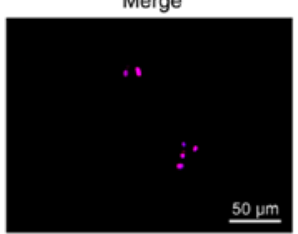

\section{E}

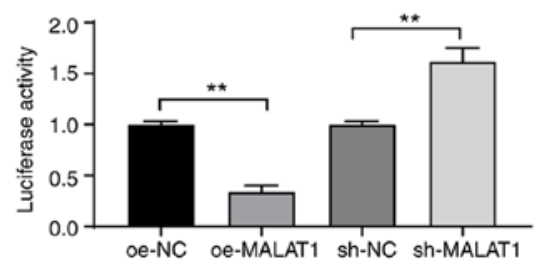

G

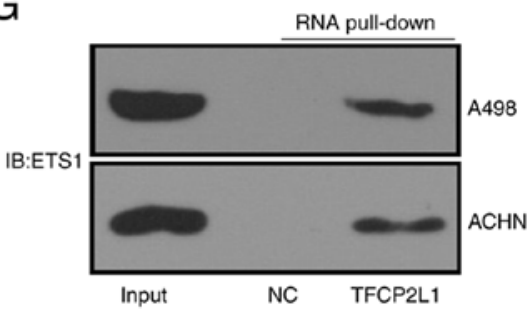

F

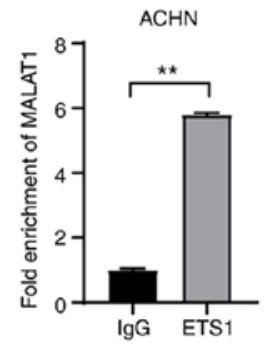

Input

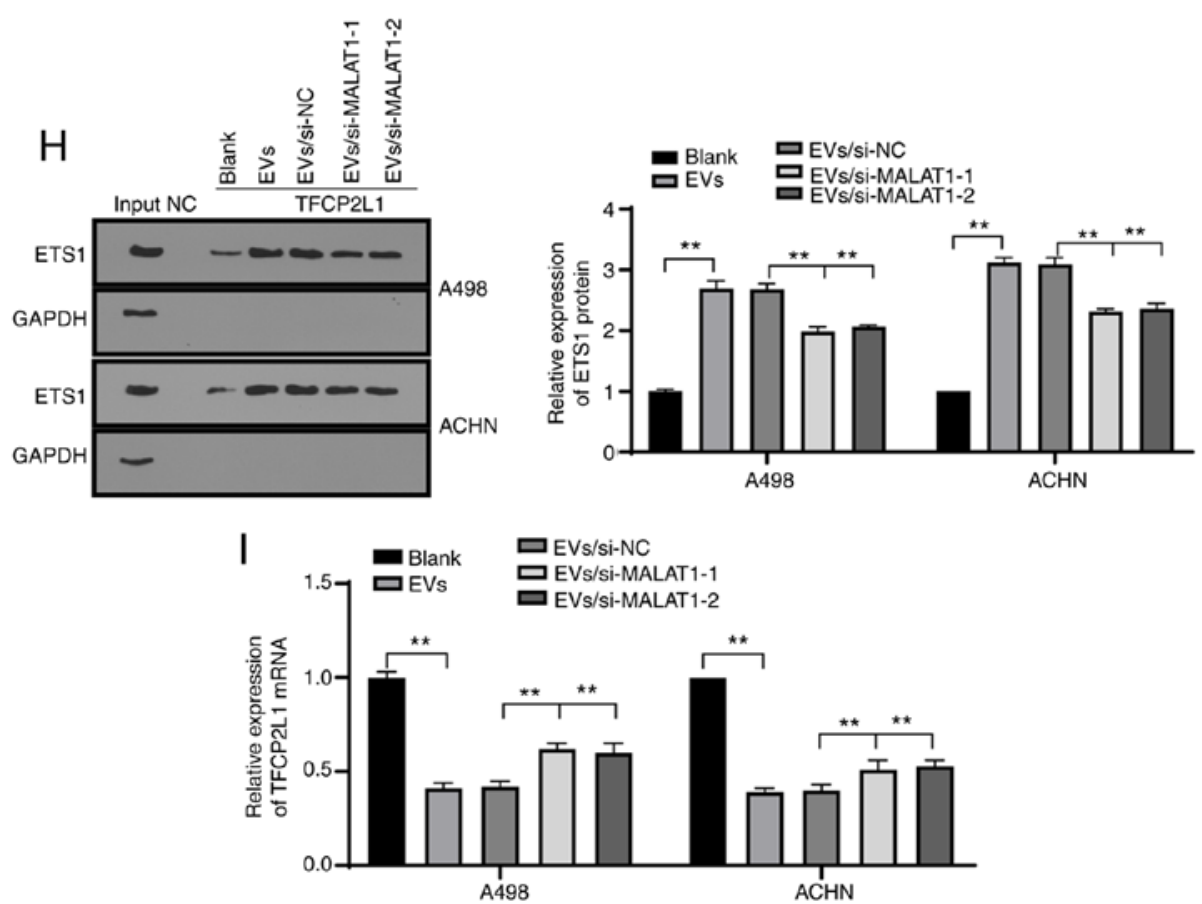

Figure 5. 786-O-EVs carry MALAT1 to regulate transcription factor ETS1 and reduce TFCP2L1 activity. (A) Prediction of MALAT1 regulatory transcription factors in KIRC and KIRP; the two circles in the figure represent the predicted transcription factors in KIRC and KIRP, and the middle part represents the intersections of the two sets of data. (B) Network diagram of interaction analysis between candidate transcription factors and genes known related to RCC; the diamonds represent the screened four candidate transcription factors, and the circles represent RCC-related genes obtained from the DisGeNET database (https://www. disgenet.org/); the darker color of the graph represents higher core degree of the gene in the network diagram and more interaction genes. (C) Prediction of ETS1 candidate target genes; the circle on the right side represents the downregulated genes in the GSE16441 chip, and the left side shows the prediction results of JASPAR database (http://jaspar.genereg.net/) on ETS1 target gene, and the middle part represents the intersection of the two groups of data. (D) FISH was used to detect subcellular localization of MALAT1. (E) Dual-luciferase reporter gene assay was used to verify the effect of MALAT1 on TFCP2L1 promoter activity. (F) RIP was used to detect the binding of MALAT1 to transcription factor ETS1. (G and H) RNA pull-down was used to detect the binding relationship between ETS1 and TFCP2L1 promoter. (I) qPCR was used to detect the mRNA expression of TFCP2L1. Each experiment was repeated three times independently. Data in panel E were analyzed using one-way ANOVA, and data in panel F were analyzed by independent t-test and data in panels $\mathrm{H}$ and I were analyzed using two-way ANOVA, followed by Tukey's multiple comparisons test. ${ }^{* *} \mathrm{P}<0.01$. EVs, extracellular vesicles; MALAT1, metastasis-associated lung adenocarcinoma transcript 1 ; RCC, renal cell carcinoma; TFCP2L1, transcription factor CP2 like 1; ETS1, ETS proto-oncogene 1, transcription factor. 
Table II. Differential expression of candidate genes in the chip GSE16441.

\begin{tabular}{lccc}
\hline Symbol & $\log \mathrm{FC}$ & P-value & adj.P-value \\
\hline TFCP2L1 & -5.698466882 & $6.02 \mathrm{E}-17$ & $3.09 \mathrm{E}-14$ \\
ESRRB & -2.343714182 & $4.84 \mathrm{E}-12$ & $3.05 \mathrm{E}-10$ \\
PLAG1 & -2.789086764 & $7.98 \mathrm{E}-12$ & $4.73 \mathrm{E}-10$ \\
KLF5 & -2.069653766 & $8.88 \mathrm{E}-10$ & $2.59 \mathrm{E}-08$ \\
TP63 & -2.593375572 & $1.91 \mathrm{E}-06$ & $1.60 \mathrm{E}-05$ \\
\hline
\end{tabular}

TFCP2L1, transcription factor CP2 like 1; ESRRB, estrogen related receptor $\beta$; PLAG1, pleomorphic adenoma gene 1; KLF5, Kruppel like factor $5 ; T P 63$, tumor protein $\mathrm{p} 63$.

(Fig. 6A). Five weeks after EV injection, the mice were euthanized, and the xenografts were dissected and weighed. The results revealed that 786-O-EV treatment apparently increased the average tumor weight (Fig. 6B) (both $\mathrm{P}<0.01$ ). In addition, immunohistochemical staining revealed that the 786-O-EVs resulted in noticeably increased Ki67-positive rates, while the tumor growth-promoting effect of 786-O-EVs was weakened after inhibition of MALAT1 in the 786-O cells (Fig. 6C) (both $\mathrm{P}<0.01)$. Moreover, the lung metastasis model was established via tail vein injection. According to $H \& E$ staining results, nude mice exhibited increased lung tissue lesions after 786-O-EV treatment, while the lung tissue lesions were reduced after silencing of MALAT1 in the 786-O cells (Fig. 6D). In conclusion, 786-O-EVs released MALAT1 to promote RCC cell growth and metastasis in vivo.

\section{Discussion}

Renal cell carcinoma (RCC) ranks as the second most prevalent cause of fatality associated with urinary tract tumors, owing to its intricate diagnosis illustrated by several asymptomatic characteristics (8). Research has solidified the vital contributions of tumor-derived extracellular vesicles (EVs) in facilitating tumor progression and metastasis via promoting pre-metastatic niche preparation, which is induced by their accommodating capacity of multiple molecules, including lncRNAs (29). In this study, our findings confirmed that 786-O-EVs promoted RCC invasion and metastasis via transport of metastasis-associated lung adenocarcinoma transcript 1 (MALAT1) to facilitate the binding of transcription factor ETS proto-oncogene 1, transcription factor (ETS1) and transcription factor $\mathrm{CP} 2$ like 1 (TFCP2L1) promoter and downregulate TFCP2L1 (Fig. 7).

Tumor-derived EVs are vital modulators for intercellular communication and essentially participate in promoting primary tumor growth and metastasis (30). As elicited by our results, after exposure to 786-O-EVs, the A498 and ACHN cells showed evidently increased cell viability, improved migration and invasion abilities, and promotion of epithelial mesenchymal transition (EMT). Consistently, an existing study flagged the significant contributions of autologous cancer cell-secreted EVs in promotion of the malignant behaviors of cancer cells (31). Tumor-derived EVs induce cancer cell invasion and metastasis, and promote EMT $(32,33)$.

Research has extensively established the ability of lncRNAs to be packaged into EVs and then transferred to the recipient cells, possessing considerable aptitude for cancer treatment (34). LncRNA MALAT1 has been indicated to serve as an oncogenic mediator in RCC with an aberrant overexpression (35). Our study documented a notably elevated MALAT1 expression in A498 and ACHN cells after treatment of 786-O-EVs, which elicited a profound reduction after silencing of MALAT1 in the 786-O cells, with membrane-encapsulated release of MALAT1 over direct release. Consistently, as evidenced by numerous studies, MALAT1 shows a predominant expression in EVs secreted by multiple cancer cells, such as thyroid cancer and epithelial ovarian cancer, which is transported to the recipient cells to manipulate the progression of cancers $(18,36)$. Altogether, $786-\mathrm{O}-\mathrm{EV}$ s can radically mediate the intercellular communication in RCC via delivery of MALAT1.

To further validate the communicative ability of 786-O-EVs of MALAT1 in RCC, we silenced the MALAT1 expression in 786-O cells to extract the EVs. Our data validated the weakening effect of MALAT1 depletion in 786-O cells on the promotive effects of 786-O-EVs on RCC cell viability, invasion and migration, as well as EMT. In consistency, numerous studies have confirmed that the high-level MALAT1 is tightly bound with enhanced viability, migration and invasion, and EMT of RCC cells (17). MALAT1 knockdown helps suppress the malignant biological behaviors of RCC cells (37). Similarly, the role of 786-O-EV-carried MALAT1 was further verified in nude mice in vivo, as evidenced by inhibited tumor growth and alleviated lung metastasis after inhibition of MALAT1 in the 786-O cells. The depletion of MALAT1 exercises inhibitory effects on tumor metastasis to vividly suppress xenograft growth in nude mice with RCC (38). Briefly, 786-O-EVs transferred MALAT1 as a facilitator of the viability, invasion and migration and EMT of RCC cells, as well as RCC tumor growth and lung metastasis in vivo.

For a comprehensive understanding of the regulatory mechanism of MALAT1, we retrieved several databases, and performed FISH, dual-luciferase reporter gene assay, RIP and RNA pull-down to identify the downstream factors. Our findings identified the ETS1 gene as a solemn regulatory transcription factor of MALAT1 alongside TFCP2L1 as the most significantly downregulated gene in the RCC expression chip GSE16441. JASPAR database supported the transcriptional regulation of TFCP2L1 by ETS1. Moreover, our data verified that the TFCP2L1 gene promoter possessed multiple binding sites with ETS1. The effect of MALAT1 on TFCP2L1 activity was verified by the dual-luciferase reporter assay, and it could be speculated that the MALAT1/ETS1 axis exerts a malignant phenotype, such as proliferation and invasiveness. As previously, ETS1 as an oncogene is identified in close association with poor survival in multiple RCC specimens (27). Elevation of ETS1 via lncRNA-mediated regulation fosters the cell malignant behaviors and tumor growth in clear cell renal cell carcinoma (ccRCC) (39). Deregulated TFCP2L1 is intimately bound with various cancers, including ccRCC (40). Furthermore, our data demonstrated that MALAT1 negatively regulated TFCP2L1 via specific binding to ETS1; 786-O-EV treatment enhanced 

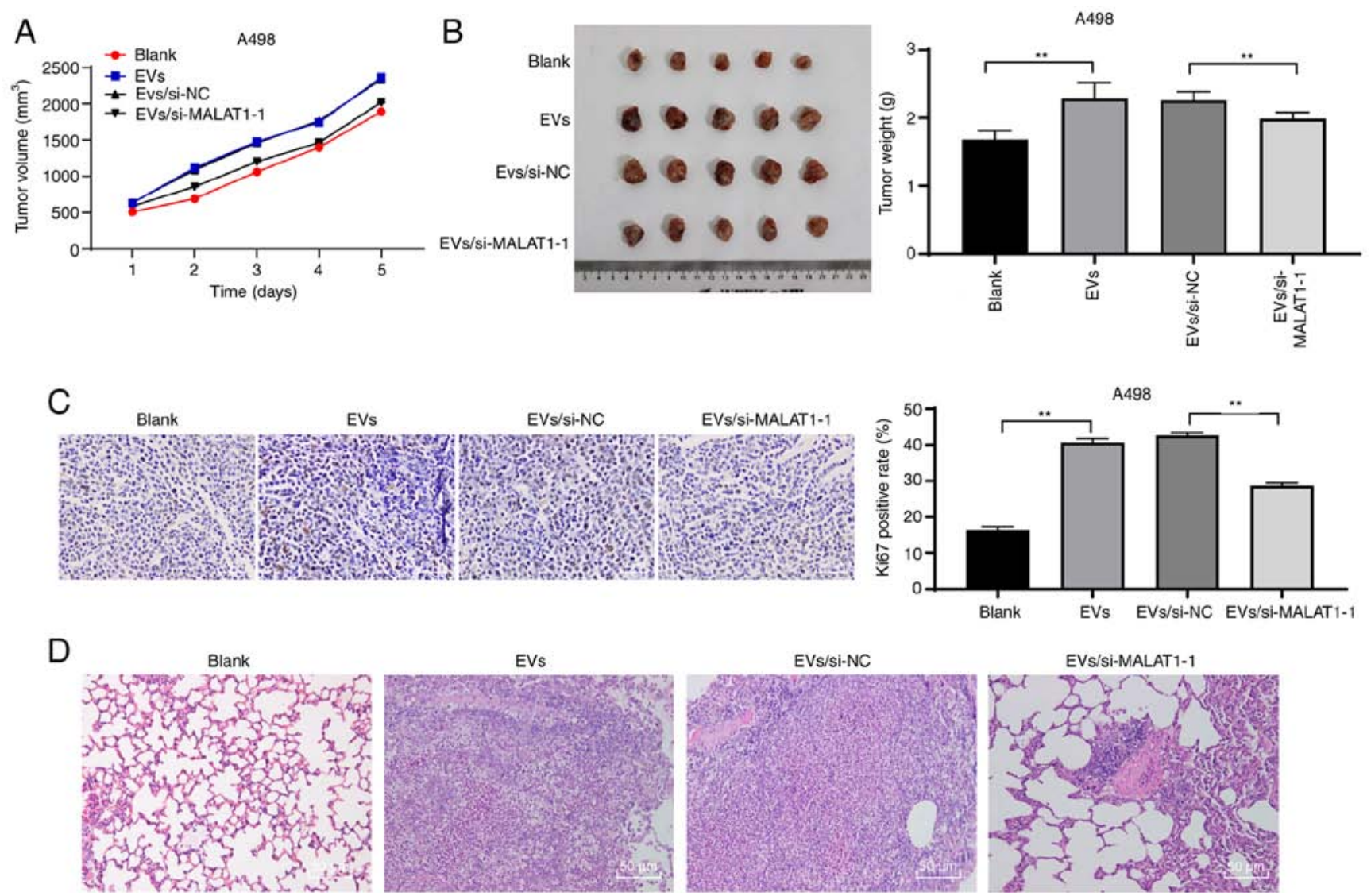

Figure 6. 786-O-EVs release MALAT1 to promote RCC cell growth and metastasis in vivo. (A) Tumor volume was measured. (B) Tumor weight was measured. (C) Immunohistochemical staining was used to detect the positive rate of Ki67. (D) H\&E staining was used to evaluate the pathological conditions of lung tissues in each group. A-D $(n=6)$. Data were analyzed using one-way ANOVA, followed by Tukey's multiple comparisons test. " $\mathrm{P}<0.01$. EVs, extracellular vesicles; MALAT1, metastasis-associated lung adenocarcinoma transcript 1; RCC, renal cell carcinoma.

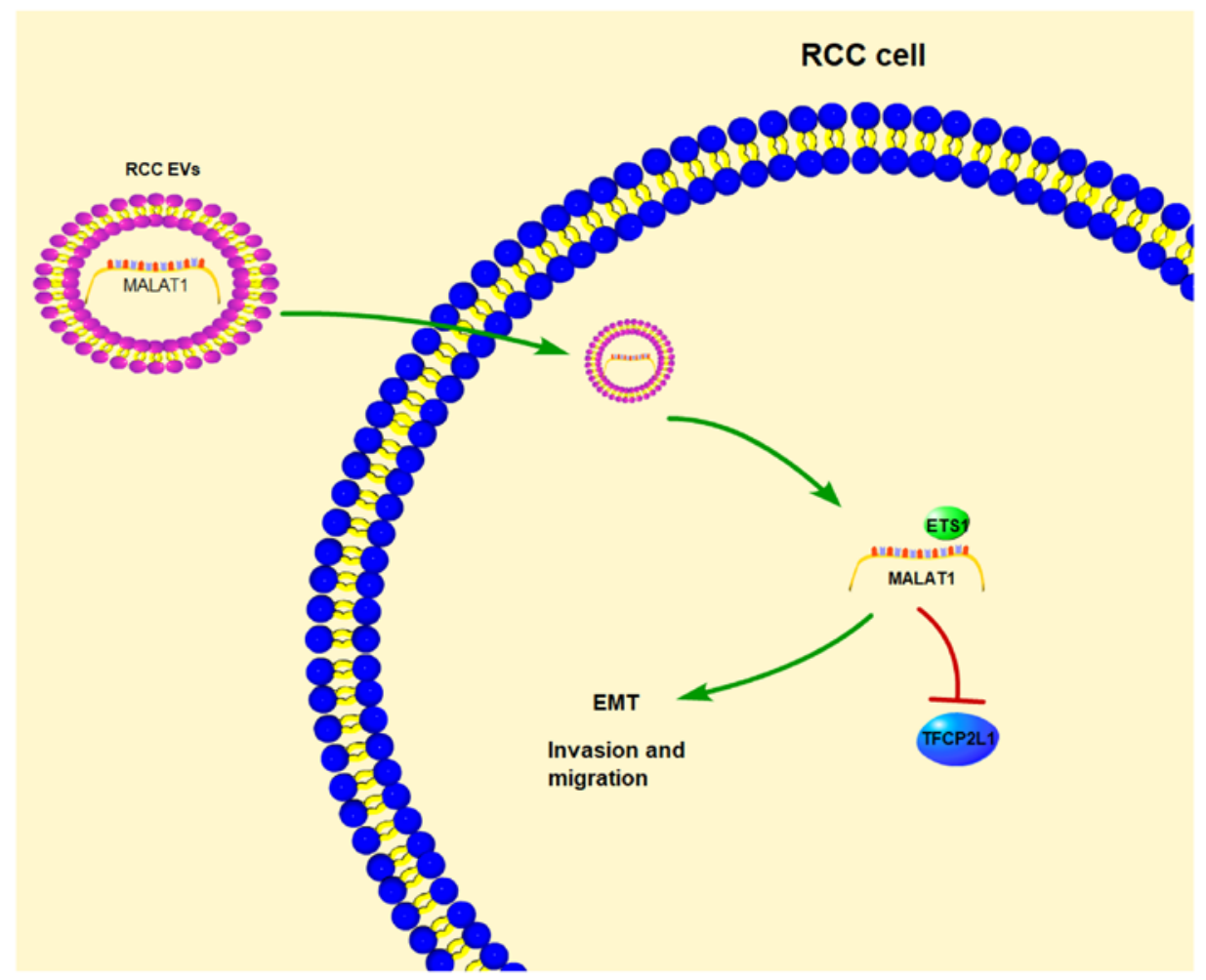

Figure 7. Mechanism model diagram. lncRNA MALAT1 carried by 786-O-EVs promotes the invasion and migration of renal cell carcinoma cells by regulating transcription factor ETS1 and affecting TFCP2L1 activity. IncRNA, long non-coding RNA; EVs, extracellular vesicles; MALAT1, metastasis-associated lung adenocarcinoma transcript 1; RCC, renal cell carcinoma; TFCP2L1, transcription factor CP2 like 1; ETS1, ETS proto-oncogene 1, transcription factor. 
the inhibition of ETS1 transcription and evidently reduced the TFCP2L1 level in RCC cells, which were reversed after knockdown of MALAT1 in the 786-O cells. LncRNAs participate in epigenetic modification via chromosome remodeling, transcriptional regulation via transcription factor modulation and post-transcriptional regulation via mRNA alternative splicing respectively (41). LncRNA CASC19, as a competitive endogenous RNA, was found to upregulate ETS1 expression by sponging miR-532 (39), a participant of post-transcriptional regulation. The current study explored the direct interaction between RNA and certain transcription factors. LncRNA MALAT1 localized to the nucleus, bound with the ETS1 protein and participate in transcriptional regulation by complex formation and localization to specific gene sequences. In consistency with the preceding finding, TFCP2L1 was proposed to be essential in normal renal development (28), with abnormal downregulation in ccRCC (42). However, the interplay between IncRNA MALAT1 and ETS1, and the relationship between ETS1 and TFCP2L1 have not been elucidated yet, which, conversely, validates the novelty of this study. Briefly, we concluded that mechanically 786-O-EVs shuttled MALAT1 to downregulate TFCP2L1 expression by promoting the binding of transcription factor ETS1 and TFCP2L1 promoter in RCC.

Collectively, our study demonstrated that 786-O-EVs promoted RCC cell invasion and metastasis via transporting MALAT1 and regulating the ETS1/TFCP2L1 axis. These results identified a novel tumor-derived EV-based therapy for RCC patients, where the development of a blockade of the MALAT1/ETS1/TFCP2L1 axis might serve as a promising therapeutic approach for RCC. Although the present study provided therapeutic value for RCC treatment, the experimental results and clinical application need further verification.

\section{Acknowledgements}

Not applicable.

\section{Funding}

No funding was received.

\section{Availability of data and materials}

The data that support the findings of this study are available from the corresponding author upon reasonable request.

\section{Authors' contributions}

CJ, LS and KL conceived and designed the study. CJ, WL, YQ, YZ, BZ, ZL, YL and QZ performed the experiments. CJ, LS and KL wrote the manuscript. CL and LS reviewed and edited the manuscript. All authors read and approved the manuscript and agree to be accountable for all aspects of the research in ensuring that the accuracy or integrity of any part of the work are appropriately investigated and resolved.

\section{Ethics approval and consent to participate}

The present study received approval from the Ethics Committee of The Second Affiliated Hospital of Harbin Medical
University (HM-2018-0524). Significant efforts were made to minimize both the number of animals and their suffering. All procedures were strictly conducted in accordance with the code of ethics.

\section{Patient consent for publication}

Not applicable.

\section{Competing interests}

The authors declared that they have no competing interests.

\section{References}

1. Hanna KS: A review of checkpoint inhibitors in the management of renal cell carcinoma. J Oncol Pharm Pract 26: 445-458, 2020.

2. Abdellah A, Selma K, Elamin M, Asmae T, Lamia R Abderrahmane M, Sanaa el M, Hanan E, Tayeb $\mathrm{K}$ and Noureddine B: Renal cell carcinoma in children: Case report and literature review. Pan Afr Med J 20: 84, 2015.

3. Abdulfatah E, Kennedy JM, Hafez K, Davenport MS, Xiao H, Weizer AZ, Palapattu GS, Morgan TM, Mannan R, Wang XM, et al: Clinicopathological characterisation of renal cell carcinoma in young adults: A contemporary update and review of literature. Histopathology 76: 875-887, 2020.

4. Gray RE and Harris GT: Renal cell carcinoma: Diagnosis and management. Am Fam Physician 99: 179-184, 2019.

5. Makhov P, Joshi S, Ghatalia P, Kutikov A, Uzzo RG and Kolenko VM: Resistance to systemic therapies in clear cell renal cell carcinoma: Mechanisms and management strategies. Mol Cancer Ther 17: 1355-1364, 2018.

6. Price M, Wu CC, Genshaft S, Sadow PM, Xie L, Shepard JO and McDermott $S$ : Imaging and management of intrathoracic renal cell carcinoma metastases. AJR Am J Roentgenol 210: 1181-1191, 2018.

7. Adibi M, Thomas AZ, Borregales LD, Matin SF, Wood CG and Karam JA: Surgical considerations for patients with metastatic renal cell carcinoma. Urol Oncol 33: 528-537, 2015.

8. Liu X, Zhang M, Liu X, Sun H, Guo Z, Tang X, Wang Z, Li J, Li H, Sun W and Zhang Y: Urine metabolomics for renal cell carcinoma (RCC) prediction: Tryptophan metabolism as an important pathway in RCC. Front Oncol 9: 663, 2019.

9. Watson DC, Bayik D, Srivatsan A, Bergamaschi C, Valentin A, Niu G, Bear J, Monninger M, Sun M, Morales-Kastresana A, et al: Efficient production and enhanced tumor delivery of engineered extracellular vesicles. Biomaterials 105: 195-205, 2016.

10. Qin Z, Xu Q, Hu H, Yu L and Zeng S: Extracellular vesicles in renal cell carcinoma: Multifaceted roles and potential applications identified by experimental and computational methods. Front Oncol 10: 724, 2020.

11. Grange C, Brossa A and Bussolati B: Extracellular vesicles and carried miRNAs in the progression of renal cell carcinoma. Int J Mol Sci 20: 1832, 2019.

12. Sheehan C and D'Souza-Schorey C: Tumor-derived extracellular vesicles: Molecular parcels that enable regulation of the immune response in cancer. J Cell Sci 132: jcs235085, 2019.

13. Ma P, Pan Y, Li W, Sun C, Liu J, Xu T and Shu Y: Extracellular vesicles-mediated noncoding RNAs transfer in cancer. J Hematol Oncol 10: 57, 2017.

14. Liu X, Hao Y, Yu W, Yang X, Luo X, Zhao J, Li J, Hu X and Li L: Long non-coding RNA emergence during renal cell carcinoma tumorigenesis. Cell Physiol Biochem 47: 735-746, 2018.

15. Zhang $Y$ and Tang L: The application of lncRNAs in cancer treatment and diagnosis. Recent Pat Anticancer Drug Discov 13: 292-301, 2018.

16. Zhao M, Wang S, Li Q, Ji Q, Guo P and Liu X: MALAT1: A long non-coding RNA highly associated with human cancers. Oncol Lett 16: 19-26, 2018.

17. Li Z, Ma Z and Xu X: Long non-coding RNA MALAT1 correlates with cell viability and mobility by targeting miR-22-3p in renal cell carcinoma via the PI3K/Akt pathway. Oncol Rep 41: $1113-1121,2019$. 
18. Qiu JJ, Lin XJ, Tang XY, Zheng TT, Lin YY and Hua KQ: Exosomal metastasis-associated lung adenocarcinoma transcript 1 promotes angiogenesis and predicts poor prognosis in epithelial ovarian cancer. Int J Biol Sci 14: 1960-1973, 2018.

19. Essandoh K, Yang L, Wang X, Huang W, Qin D, Hao J, Wang Y, Zingarelli B, Peng T and Fan GC: Blockade of exosome generation with GW4869 dampens the sepsis-induced inflammation and cardiac dysfunction. Biochim Biophys Acta 1852: 2362-2371, 2015

20. Liu H, Brannon AR, Reddy AR, Alexe G, Seiler MW, Arreola A, Oza JH, Yao M, Juan D, Liou LS, et al: Identifying mRNA targets of microRNA dysregulated in cancer: With application to clear cell renal cell carcinoma. BMC Syst Biol 4: 51, 2010.

21. Ritchie ME, Phipson B, Wu D, Hu Y, Law CW, Shi W and Smyth GK: limma powers differential expression analyses for RNA-sequencing and microarray studies. Nucleic Acids Res 43: e47, 2015.

22. Wei JX, Lv LH, Wan YL, Cao Y, Li GL, Lin HM, Zhou R, Shang CZ, Cao J, He H, et al: Vps4A functions as a tumor suppressor by regulating the secretion and uptake of exosomal microRNAs in human hepatoma cells. Hepatology 61: 1284-1294, 2015.

23. Livak KJ and Schmittgen TD: Analysis of relative gene expression data using real-time quantitative PCR and the 2(-Delta Delta C(T)) method. Methods 25: 402-408, 2001

24. Yeon JH, Jeong HE, Seo H, Cho S, Kim K, Na D, Chung S, Park J, Choi N and Kang JY: Cancer-derived exosomes trigger endothelial to mesenchymal transition followed by the induction of cancer-associated fibroblasts. Acta Biomater 76: 146-153, 2018.

25. Pan L, Liang W, Fu M, Huang ZH, Li X, Zhang W, Zhang P, Qian H, Jiang PC, Xu WR and Zhang X: Exosomes-mediated transfer of long noncoding RNA ZFAS1 promotes gastric cancer progression. J Cancer Res Clin Oncol 143: 991-1004, 2017.

26. Zhang H, Li W, Gu W, Yan Y, Yao X and Zheng J: MALAT1 accelerates the development and progression of renal cell carcinoma by decreasing the expression of miR-203 and promoting the expression of BIRC5. Cell Prolif 52: e12640, 2019.

27. Zhai W, Ma J, Zhu R, Xu C, Zhang J, Chen Y, Chen Z, Gong D, Zheng J, Chen C, et al: MiR-532-5p suppresses renal cancer cell proliferation by disrupting the ETS1-mediated positive feedback loop with the KRAS-NAP1L1/P-ERK axis. Br J Cancer 119: 591-604, 2018

28. Tun HW, Marlow LA, von Roemeling CA, Cooper SJ, Kreinest P, Wu K, Luxon BA, Sinha M, Anastasiadis PZ and Copland JA: Pathway signature and cellular differentiation in clear cell renal cell carcinoma. PLoS One 5: e10696, 2010.

29. Xu R, Rai A, Chen M, Suwakulsiri W, Greening DW and Simpson RJ: Extracellular vesicles in cancer-implications for future improvements in cancer care. Nat Rev Clin Oncol 15: $617-638,2018$

30. Becker A, Thakur BK, Weiss JM, Kim HS, Peinado H and Lyden D: Extracellular vesicles in cancer: Cell-to-cell mediators of metastasis. Cancer Cell 30: 836-848, 2016.
31. Li YJ, Wu JY, Hu XB, Wang JM and Xiang DX: Autologous cancer cell-derived extracellular vesicles as drug-delivery systems: A systematic review of preclinical and clinical findings and translational implications. Nanomedicine (Lond) 14: 493-509, 2019.

32. Chen J, Fei X, Wang J and Cai Z: Tumor-derived extracellular vesicles: Regulators of tumor microenvironment and the enlightenment in tumor therapy. Pharmacol Res 159: 105041, 2020.

33. Syn N, Wang L, Sethi G, Thiery JP and Goh BC Exosome-mediated metastasis: From epithelial-mesenchymal transition to escape from immunosurveillance. Trends Pharmacol Sci 37: 606-617, 2016

34. Li Z, Zhu X and Huang S: Extracellular vesicle long non-coding RNAs and circular RNAs: Biology, functions and applications in cancer. Cancer Lett 489: 111-120, 2020.

35. Hirata H, Hinoda Y, Shahryari V, Deng G, Nakajima K, Tabatabai ZL, Ishii N and Dahiya R: Long noncoding RNA MALAT1 promotes aggressive renal cell carcinoma through Ezh2 and interacts with miR-205. Cancer Res 75: 1322-1331, 2015.

36. Hardin H, Helein H, Meyer K, Robertson S, Zhang R, Zhong W and Lloyd RV: Thyroid cancer stem-like cell exosomes: Regulation of EMT via transfer of IncRNAs. Lab Invest 98: 1133-1142, 2018.

37. Jiang LT, Wan $\mathrm{CH}$, Guo QH, Yang SJ, Wu JD and Cai J: Long noncoding RNA metastasis-associated lung adenocarcinoma transcript 1 (MALAT1) promotes renal cell carcinoma progression via sponging miRNA-429. Med Sci Monit 24: 1794-1801, 2018.

38. Chen S, Ma P, Zhao Y, Li B, Jiang S, Xiong H, Wang Z, Wang H, Jin X and Liu C: Biological function and mechanism of MALAT-1 in renal cell carcinoma proliferation and apoptosis: role of the MALAT-1-Livin protein interaction. J Physiol Sci 67: 577-585, 2017.

39. Luo Y,Liu F, Yan C, Qu W,Zhu L, GuoZ,Zhou Fand Zhang W: Long non-coding RNA CASC19 sponges microRNA-532 and promotes oncogenicity of clear cell renal cell carcinoma by increasing ETS 1 expression. Cancer Manag Res 12: 2195-2207, 2020.

40. Kotarba G, Krzywinska E, Grabowska AI, Taracha A and Wilanowski T: TFCP2/TFCP2L1/UBP1 transcription factors in cancer. Cancer Lett 420: 72-79, 2018.

41. Fanelli GN, Gasparini P, Coati I, Cui R, Pakula H, Chowdhury B, Valeri N, Loupakis F, Kupcinskas J, Cappellesso R and Fassan M: long-noncoding RNAs in gastroesophageal cancers. Noncoding RNA Res 3: 195-212, 2018.

42. Zaravinos A, Lambrou GI, Mourmouras N, Katafygiotis P, Papagregoriou G, Giannikou K, Delakas D and Deltas C: New miRNA profiles accurately distinguish renal cell carcinomas and upper tract urothelial carcinomas from the normal kidney. PLoS One 9: e91646, 2014.

(i) $\Theta$ This work is licensed under a Creative Commons Attribution-NonCommercial-NoDerivatives 4.0 International (CC BY-NC-ND 4.0) License. 